\title{
Monitoramento da Composição Específica da Comunidade de MACRÓfITAS AQUÁticas NO RESERVATÓRIO LUIS EdUARDO MAGALHÃES ${ }^{1}$
}

\author{
Monitoring the Specific Composition of Macrophyte Communities in Luis Eduardo Magalhaes \\ Reservoir
}

LOLIS, S.F. ${ }^{2}$ e THOMAZ, S.M. ${ }^{3}$

\begin{abstract}
RESUMO - O objetivo deste trabalho foi avaliar a composição e distribuição espacial e temporal da comunidade de macrófitas aquáticas diante de algumas variáveis abióticas no reservatório Luis Eduardo Magalhães, localizado no rio Tocantins. As coletas foram bimestrais, no periodo de março de 2005 a janeiro de 2006, em cinco regiões. Em cada região foram demarcados 15 pontos de coleta. Foram mensuradas a transparência da água e a condutividade elétrica em cada ponto de amostragem. Para avaliar a composição da comunidade e a distribuição das espécies, foi percorrida uma distância de cerca de $100 \mathrm{~m}$ de margem com embarcação em baixa velocidade, por aproximadamente 10 minutos. As espécies submersas foram inventariadas com um rastelo até $4 \mathrm{~m}$ de profundidade. Foi calculada a frequência de ocorrência e a diversidade beta. Os dados de presença e ausência das espécies foram analisados utilizando Análise de Correspondência Destendenciada - DCA. Uma ANOVA One-Way foi empregada para avaliar o efeito dos períodos e regiões sobre as variáveis abióticas e bióticas. Foi aplicado o teste a posteriori de Tukey quando houve diferenças significativas entre as variáveis analisadas $(\mathrm{P}<0,05)$. Foi registrado um total de 50 espécies. As espécies com maior frequência foram Salvinia auriculata, Oxycaryum cubense e Najas microcarpa (submersa). A maior riqueza de espécies foi registrada nas regiões fluviais do reservatório (Areias e Tocantins). Os resultados evidenciam, ainda, que a variação espacial na composição das assembleias foi mais acentuada que a variação temporal.
\end{abstract}

Palavras-chave: diversidade beta, distribuição temporal, riqueza de espécies.

\begin{abstract}
This study aimed to evaluate the composition and spatial and temporal distribution of a community of macrophytes in response to abiotic factors in Luis Eduardo Magalhães Reservoir, in the Tocantins River. Samplings were collected every two months, from March 2005 to January 2006 in five regions of the reservoir. In each region, 15 collection sites were selected. The abiotic variables measured were the Secchi disk depth, electrical conductivity, air temperature, and rain and water levels. A standardized sampling effort was applied to assess macrophyte species distribution and community composition. In each site, a distance of $100 \mathrm{~m}$ from the shore was covered with a boat maintaining low and constant velocity, for approximately 10 minutes. The submerged species were inventoried with a rake attached to a $4 m$ pipe. Incidence data were analyzed using DCA. One-Way ANOVA was applied to evaluate the effects of the periods and regions on the abiotic and biotic variables. The Tukey test a posteriori was applied when significant differences $(P<0.05)$ Checar se seria $P$ maiusculo between the variables were found. A total of 50 species was recorded. The most frequent species were Salvinia auriculata, Oxycaryum cubense and Najas microcarpa (submerged). The highest species richness was observed in the riverine regions of the reservoir (Areias and Tocantins). Our results also showed that the spatial variation in community composition was more accentuated than temporal variation.
\end{abstract}

Keywords: beta diversity, temporal distribution, species richnes.

Recebido para publicação em 5.2.2011 e na forma revisada em 6.5.2011.

2 Docente, Curso de Ciências Biológicas, Universidade Federal do Tocantins - UFT, Campus de Porto Nacional, Rua 07, Quadra 15, s/n, Jardim dos Ipês, 77500-000 Porto Nacional-TO, Brasil, <lolis.solange@gmail.com>; ${ }^{3}$ Docente, Departamento de Biologia, Núcleo de Pesquisas em Limnologia, Ictiologia e Aqüicultura, Universidade Estadual de Maringá - UEM, Av. Colombo 5790, 87020900 Maringá-PR, Brazil. 


\section{INTRODUÇÃO}

A formação de um reservatório resulta na criação de um ambiente propício para a colonização de macrófitas aquáticas de diferentes tipos biológicos (Bianchini Jr., 2003). Alguns estudos enfocam a distribuição e os fatores ecológicos associados à presença e crescimento de plantas aquáticas em reservatórios brasileiros (Bini et al., 1999, 2005; Tanaka et al., 2002; Carvalho et al., 2003; Martins et al., 2003), mas poucos foram realizados nas fases iniciais de formação do reservatório, quando este efetivamente começa a ser colonizado pelas plantas aquáticas (De Filippo, 2003). Em estudos realizados em reservatórios brasileiros de grande porte, situados na região tropical, como Tucuruí e Balbina, observou-se a colonização de espécies flutuantes livres e a expansão de suas populações nos primeiros anos do reservatório, em função da eutrofização do corpo d'água (De Filippo, 2003; Bianchini Jr., 2003). Durante a formação do reservatório de Tucuruí, por exemplo, uma grande extensão de florestas foi alagada, resultando em incremento de matéria orgânica e, consequentemente, aumento das concentrações dos nutrientes, o que provocou rápida colonização por formas flutuantes.

Em reservatórios recém-formados, durante a fase de enchimento e nos primeiros anos de operação, a invasão de plantas aquáticas flutuantes tem sido atribuída à disponibilidade de nutrientes, que caracteriza a fase inicial e oferece condições favoráveis à sua proliferação (Agostinho \& Gomes, 1997; De Filippo, 2003). No entanto, Neiff et al. (2000) discutem a ausência de uma relação direta entre a presença de macrófitas e a disponibilidade de nutrientes na água. Além destes, Rubim \& Camargo (2001) avaliaram o crescimento de $S$. molesta em um braço do rio Preto, em Itanhaém (SP), e observaram que mesmo em baixas concentrações de nutrientes essa espécie prolifera rapidamente. Esses autores concluíram que a temperatura elevada e constante favoreceu o crescimento dessa espécie.

No reservatório de Guri (Venezuela), a colonização por plantas aquáticas somente ocorreu na segunda etapa de enchimento, quando grandes áreas de floresta foram inundadas (Vilarrubia \& Cova, 1993). Nos reservatórios localizados a jusante e a montante do reservatório Lajeado, como Tucuruí e Serra da Mesa, também foram observadas infestações por S. auriculata (Junk \& Melo, 1990; De Fellipo, 2003).

A colonização por plantas aquáticas em um ambiente hídrico é uma indicação de que a água pode apresentar alterações, como, por exemplo, a eutrofização (Patton \& Starnes, 1970). Esta tem como origem a intensificação de atividades antrópicas nas bacias hidrográficas e normalmente encontra-se associada ao aporte de nutrientes para o rio, através de despejos domésticos, industriais e fertilizantes químicos empregados nos cultivos (Valente et al., 1997). A alteração do nível de água também afeta as assembleias de macrófitas aquáticas, porém os efeitos desse processo variam conforme o tipo biológico, o que depende também da amplitude e duração dessa variação (Thomaz, 2003).

Este trabalho teve por objetivo avaliar a riqueza de espécies e a composição das assembleias de macrófitas aquáticas, assim como a distribuição espacial e temporal das espécies de macrófitas aquáticas no reservatório de Luis Eduardo Magalhães. Alguns fatores abióticos foram utilizados para explicar a distribuição das espécies de macrófitas no reservatório.

\section{MATERIAL E MÉTODOS}

\section{Área de Estudo}

A bacia do rio Tocantins possui área de drenagem de $767.000 \mathrm{~km}^{2}$ e é inteiramente localizada em território brasileiro. A maioria dos afluentes apresenta baixo volume hídrico na estação seca, sem grandes áreas marginais inundáveis, e com formação de enchentes de resposta rápida às variações pluviométricas (Welcomme, 1979).

O rio Tocantins tem seu curso no sentido sul-norte, sendo formado pela conjunção dos rios Paranã e Maranhão. O rio percorre $2.500 \mathrm{~km}$ através dos Estados de Goiás, Tocantins, Maranhão e Pará, onde deságua próximo ao estuário do rio Amazonas (Paiva, 1982).

O periodo de chuvas se estende de outubro a abril, e o de seca, de maio a setembro, com 
as menores precipitações registradas em setembro (Ribeiro et al., 1995).

O reservatório Luis Eduardo Magalhães (Lajeado) está localizado no rio Tocantins (Paiva, 1982), entre os municipios de Miracema do Tocantins e Brejinho de Nazaré. O represamento apresenta extensão de $172 \mathrm{~km}$, área superficial de $626 \mathrm{~km}^{2}$, área de drenagem de $184.219,00 \mathrm{~km}^{2}$, nivel a montante de $212 \mathrm{~m} \mathrm{e}$ a jusante de $175,80 \mathrm{~m}$. O reservatório foi concluído em outubro de 2000 e o enchimento completou-se em fevereiro de 2001; opera como fio d'água e a oscilação do nível está relacionada à operação da própria usina e de outros represamentos localizados a montante.

\section{Amostragem e levantamento de dados}

Os bancos de macrófitas aquáticas avaliados foram selecionados e georreferenciados com aparelho GPS (Global Positioning System) em quatro braços do reservatório (Santa Luzia, Mangues, Água Suja e Areias) e em um trecho entre a região fluvial e intermediária do corpo principal (rio Tocantins) do reservatório (Figura 1). O intervalo entre coletas foi bimestral (março de 2005 a janeiro de 2006), e em cada região estudada foram demarcados 15 pontos de amostragem, totalizando 75 .

A transparência da água (disco de Secchi; $\mathrm{m})$ e a condutividade elétrica (medidor digital Digimed; $\mu \mathrm{S} \mathrm{cm}^{-1}$ ) foram medidas no momento da coleta. Os dados referentes a temperatura do $\operatorname{ar}\left({ }^{\circ} \mathrm{C}\right)$, precipitação $(\mathrm{mm})$ e cota altimétrica do reservatório $(\mathrm{m})$ foram obtidos da estação meteorológica localizada próximo à barragem, no municipio de Lajeado (TO).

O inventário e a avaliação da distribuição espacial e temporal das espécies no reservatório foram realizados utilizando-se um esforço amostral padronizado. Em cada ponto de amostragem, percorreram-se cerca de $100 \mathrm{~m}$ de margem com uma embarcação em baixa velocidade. O levantamento de espécies submersas foi realizado até uma profundidade de $4 \mathrm{~m}$, utilizando para tal função um rastelo.

\section{Análise dos dados}

A frequência de ocorrência de cada espécie (Fi) foi calculada para cada região estudada,

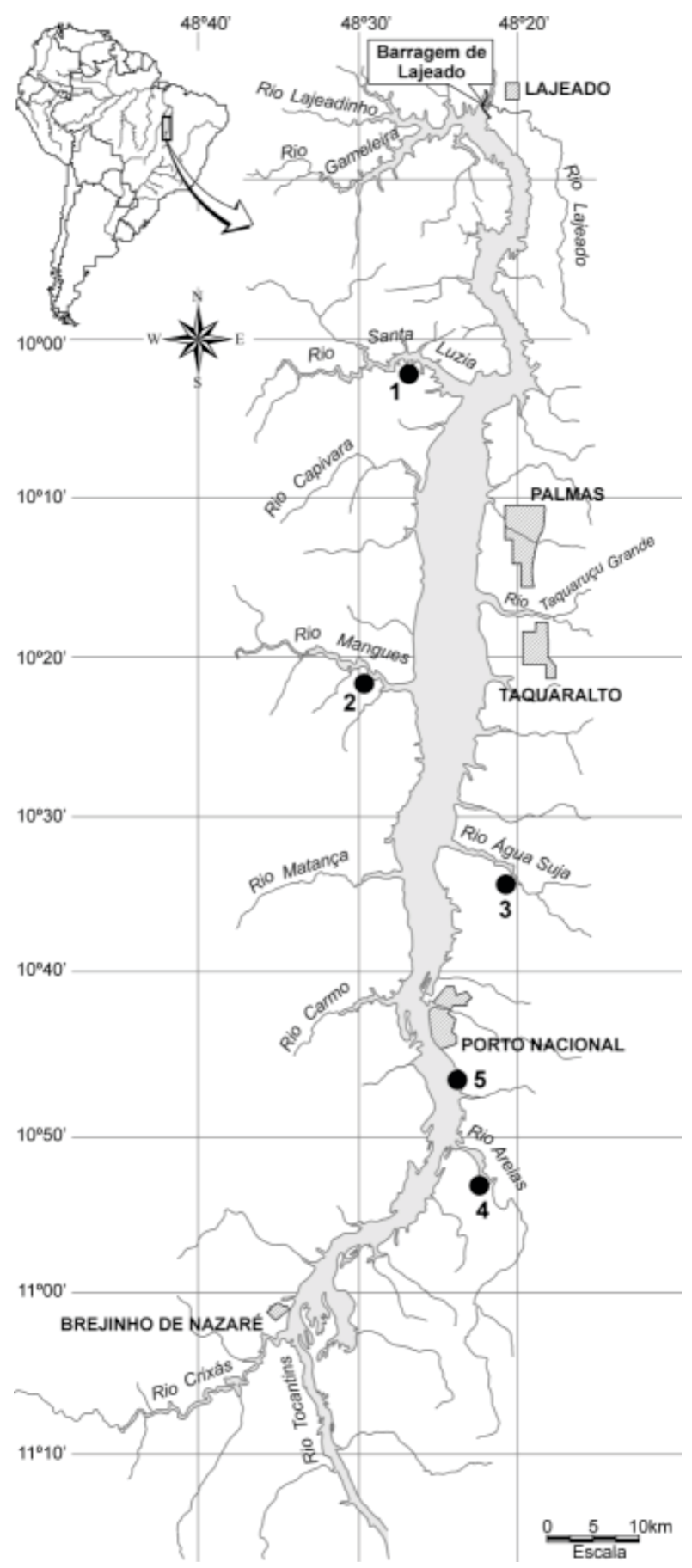

1= Santa Luzia; 2= Mangues; 3= Água Suja; 5= Tocantins; e 4= Areias.

Figura 1 - Regiões de estudo no reservatório Luis Eduardo Magalhães, rio Tocantins.

representando o número de vezes em que a espécie ocorreu em relação ao total de ocorrências. 
O indice de diversidade beta- 1 foi calculado dentro de cada região estudada em todas as épocas amostradas, com o objetivo de medir alterações na composição específica das macrófitas aquáticas (Wilson \& Shimida, 1984; Harrison et al., 1992).

Padrões de composição de espécies (presença e ausência) nas cinco regiões foram sumarizados com uma Análise de Correspondência com remoção do efeito do arco (DCA), conferindo menor peso para espécies raras. A DCA foi executada usando o programa PCORD v.3.15 para Windows (McCune \& Mefford, 1997).

Análises de variância unifatoriais foram empregadas com o objetivo de testar separadamente o efeito do período (meses) e regiões (Santa Luzia, Mangues, Água Suja, Areias e Tocantins) sobre as variáveis abióticas (condutividade e transparência) e bióticas (riqueza de espécies, diversidade beta- 1 e os dois primeiros eixos da DCA). O teste post-hoc de Tukey foi aplicado para investigar diferenças entre as médias, quando houve influência significativa dos fatores analisados $(\alpha<0,05)$. Os testes foram executados através do programa STATISTICA v. 6.0.

\section{RESULTADOS}

\section{Variáveis abióticas}

A temperatura do ar variou de $26^{\circ} \mathrm{C}$ (fevereiro) a $32{ }^{\circ} \mathrm{C}$ (setembro), com valor médio de $27^{\circ} \mathrm{C}$ durante o período de coleta (Figura 2A). Os meses de inverno foram os mais secos. A chuva foi ausente no período de junho a novembro, e os maiores valores médios de precipitação pluvial foram registrados nos meses de março a maio de 2005 e fevereiro 2006 (Figura 2B). Em geral, os maiores niveis de precipitação ocorreram nos meses de menor temperatura (Figura 2A, B). A cota altimétrica do reservatório permaneceu relativamente constante durante o período de amostragem, oscilando, em geral, menos de 0,5 m (Figura 2C).

O menor valor médio da condutividade elétrica foi registrado em Mangues $\left(54,2 \mu \mathrm{S} \mathrm{cm}^{-1}\right.$; $\mathrm{DP}=23,42)$, e o maior, em Santa Luzia $\left(79,19 \mu \mathrm{S} \mathrm{cm}^{-1}\right.$; DP=57,88). A condutividade elétrica foi significativamente diferente entre as regiões $(\mathrm{F}(4,445)$ 9,35; $\mathrm{P}=0,001)$ (Figura $3 \mathrm{~A})$. $\mathrm{Em}$ todas as regiões estudadas, a variação temporal da condutividade elétrica apresentou diferenças significativas $(F(4,444)=34,22$; $\mathrm{P}=0,001$ ) (Figura 3B-F).
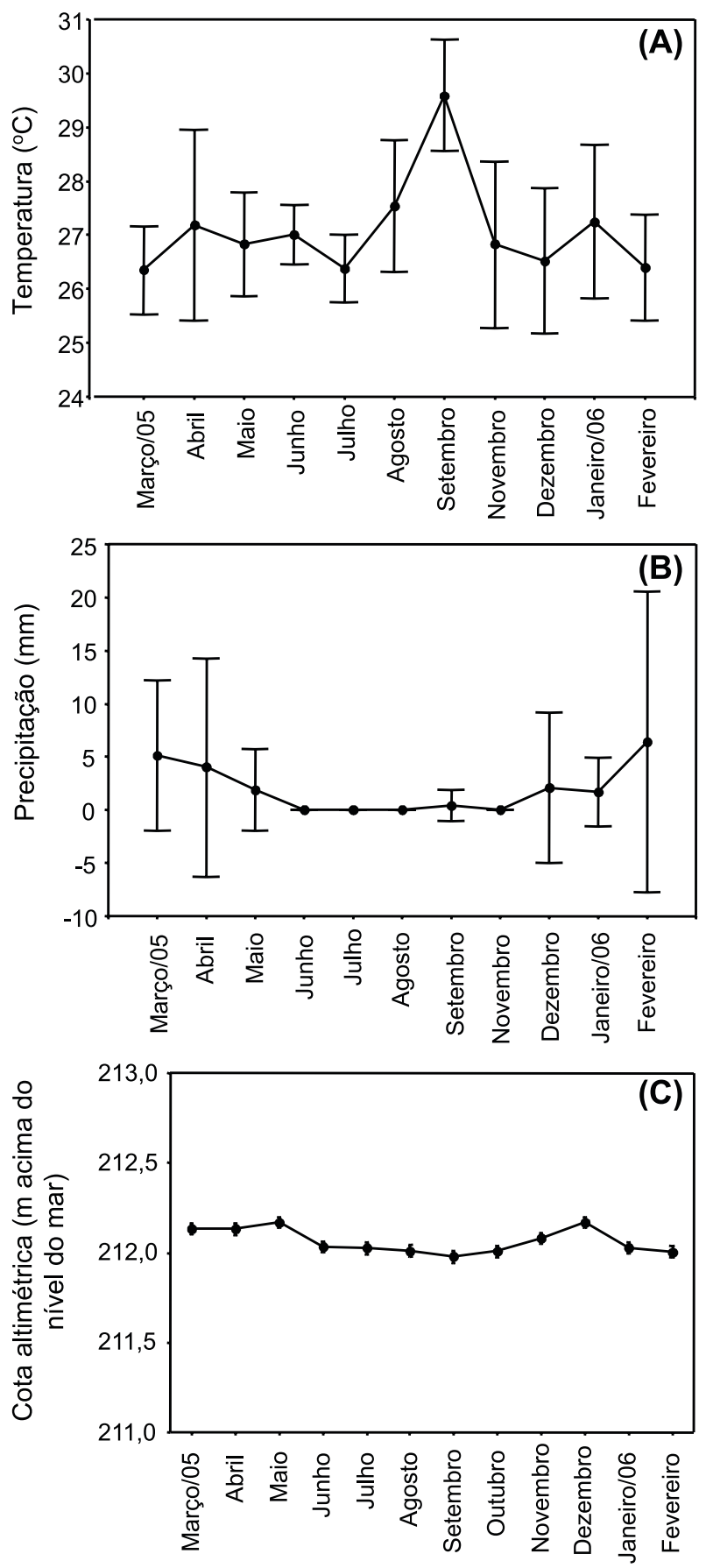

Figura 2 - Valores médios mensais e desvio-padrão para (A) temperatura do ar; (B) precipitação acumulada nos diferentes meses de avaliação; e (C) cota altimétrica do reservatório de Lajeado. 

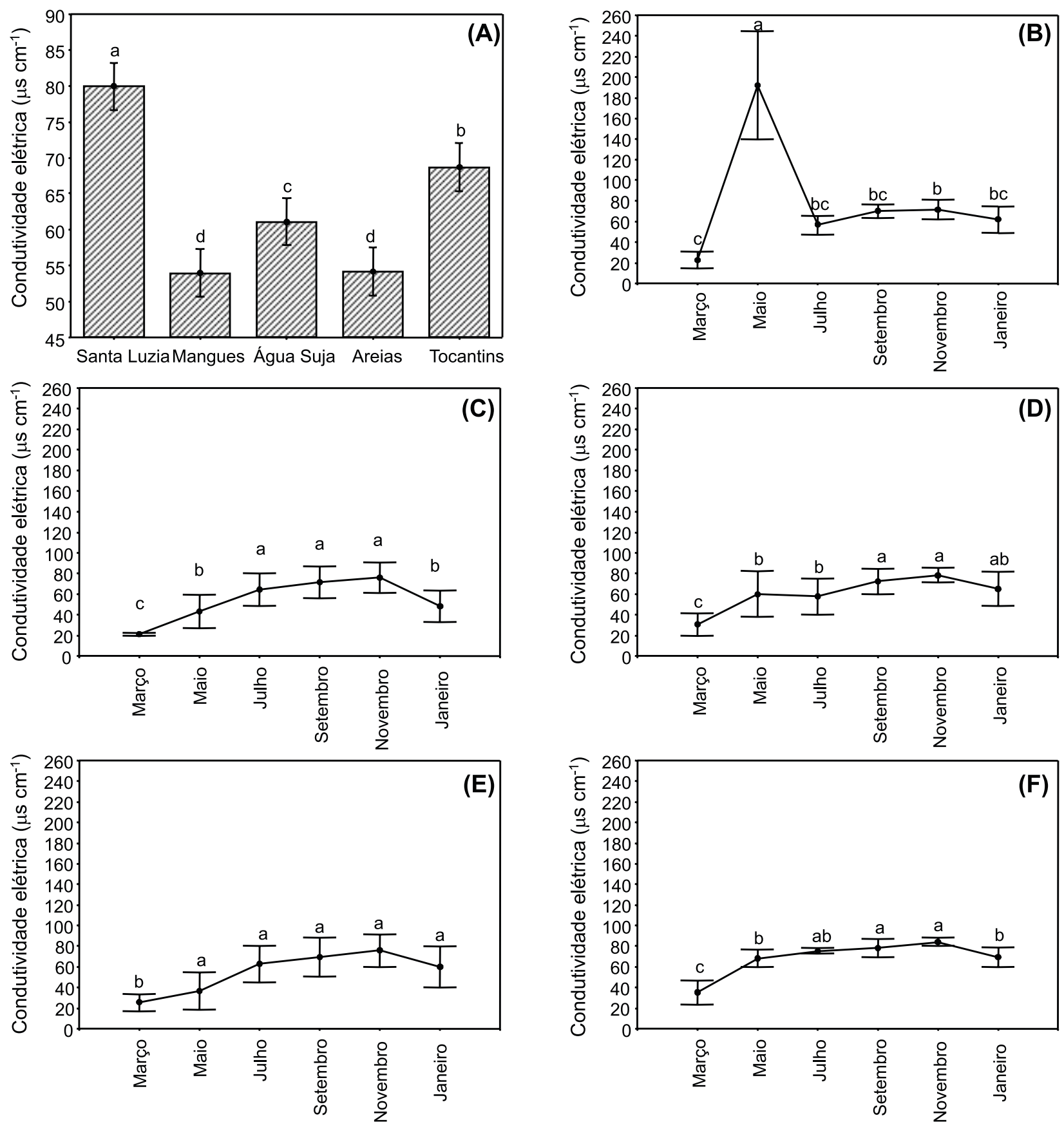

Letras iguais indicam ausência de diferenças estatísticas (Tukey; $\mathrm{P}>0,05$ ).

Figura 3 - Valores médios e desvio-padrão de condutividade elétrica nas cinco regiões do reservatório (A) e variação mensal nas regiões de Santa Luzia (B), Mangues (C), Água Suja (D), Areias (E) e Tocantins (F).

Os valores de transparência da coluna da água oscilaram entre $0,28 \mathrm{~m}(\mathrm{DP}=0,43)$ na região de Areias e 4,78 m $(\mathrm{DP}=0,96)$ na região de Mangues. Entre as regiões, a transparência da água apresentou diferenças significativas
$(F(4,445)=27,46 ; P=0,001)$ (Figura 4A), o mesmo ocorrendo para os meses de coleta $(\mathrm{F}(4$, 444) $=50,44 ; \mathrm{P}=0,001$ ) (Figura 4B a F). Em geral, os maiores valores de transparência foram registrados de maio a setembro (período de 
seca), e os menores, em março (período chuvoso). O aumento da condutividade elétrica na região de Santa Luzia coincidiu com a maior transparência da coluna de água (Figuras 3B e 4B).
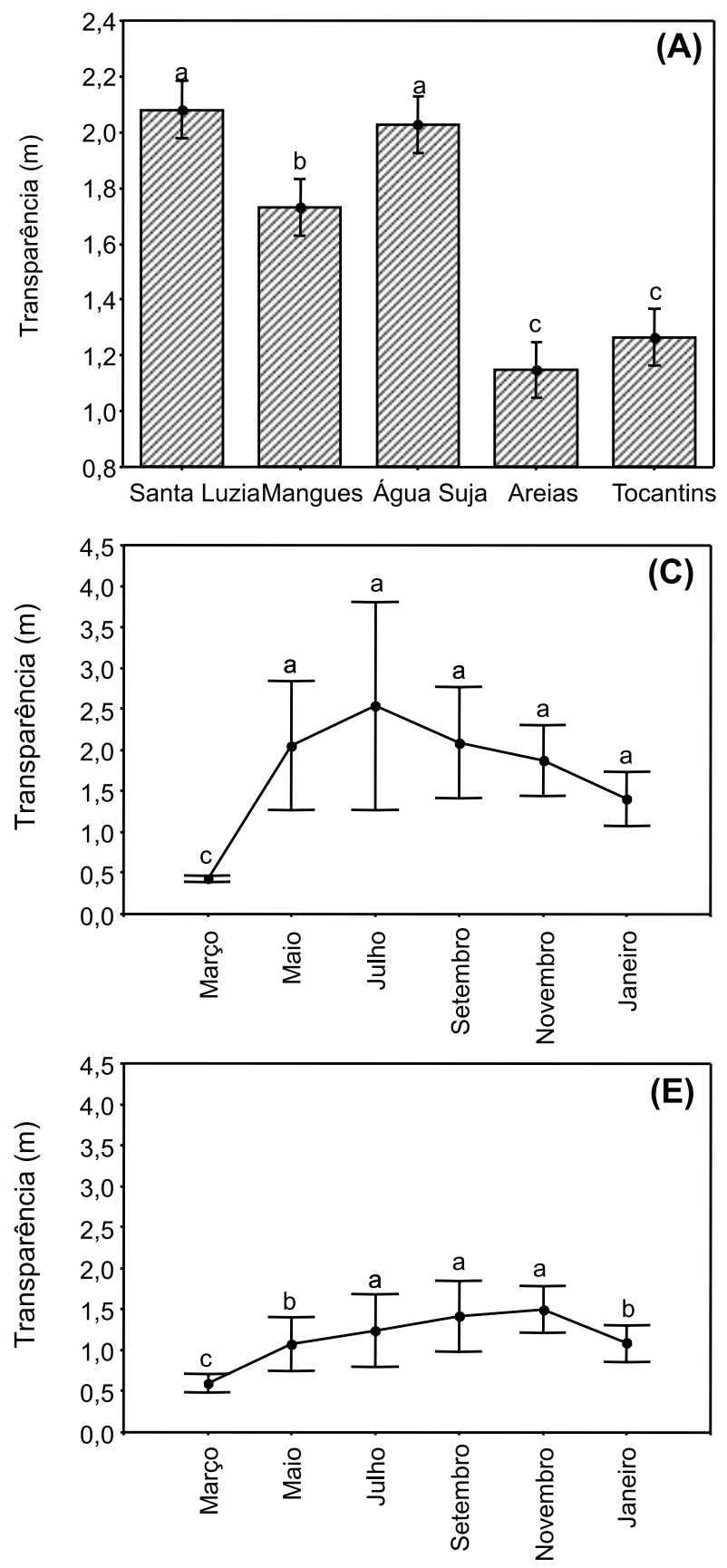

\section{Macrófitas aquáticas}

A riqueza de espécies registrada em todas as regiões de amostragem ao longo do período de estudo compreendeu um total de 50 táxons,
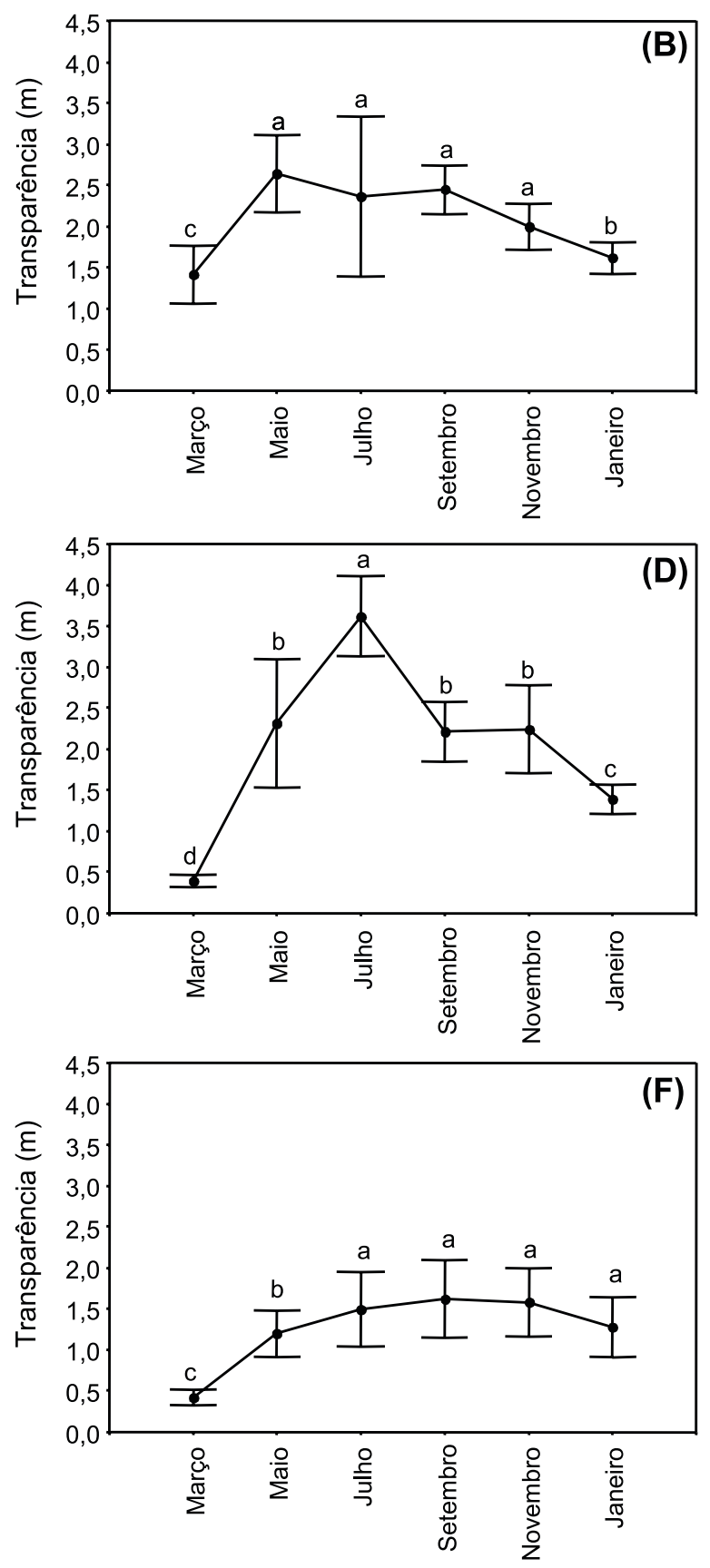

Letras iguais indicam ausência de diferenças significativas (Tukey; $\mathrm{P}>0,05$ )

Figura 4 - Valores médios e desvio-padrão da profundidade do disco de Secchi nas cinco regiões do reservatório (A) e variação mensal nas regiões de Santa Luzia (B), Mangues (C), Água Suja (D), Areias (E) e Tocantins (F). 
incluindo todos os grupos ecológicos de macrófitas aquáticas.

Entre as espécies de plantas aquáticas encontradas neste estudo, Salvinia auriculata apresentou a maior frequência de ocorrência (> 75\% em todas as regiões estudadas). Oxycaryum cubense, com frequência menor que $25 \%$ somente na região do Santa Luzia, e
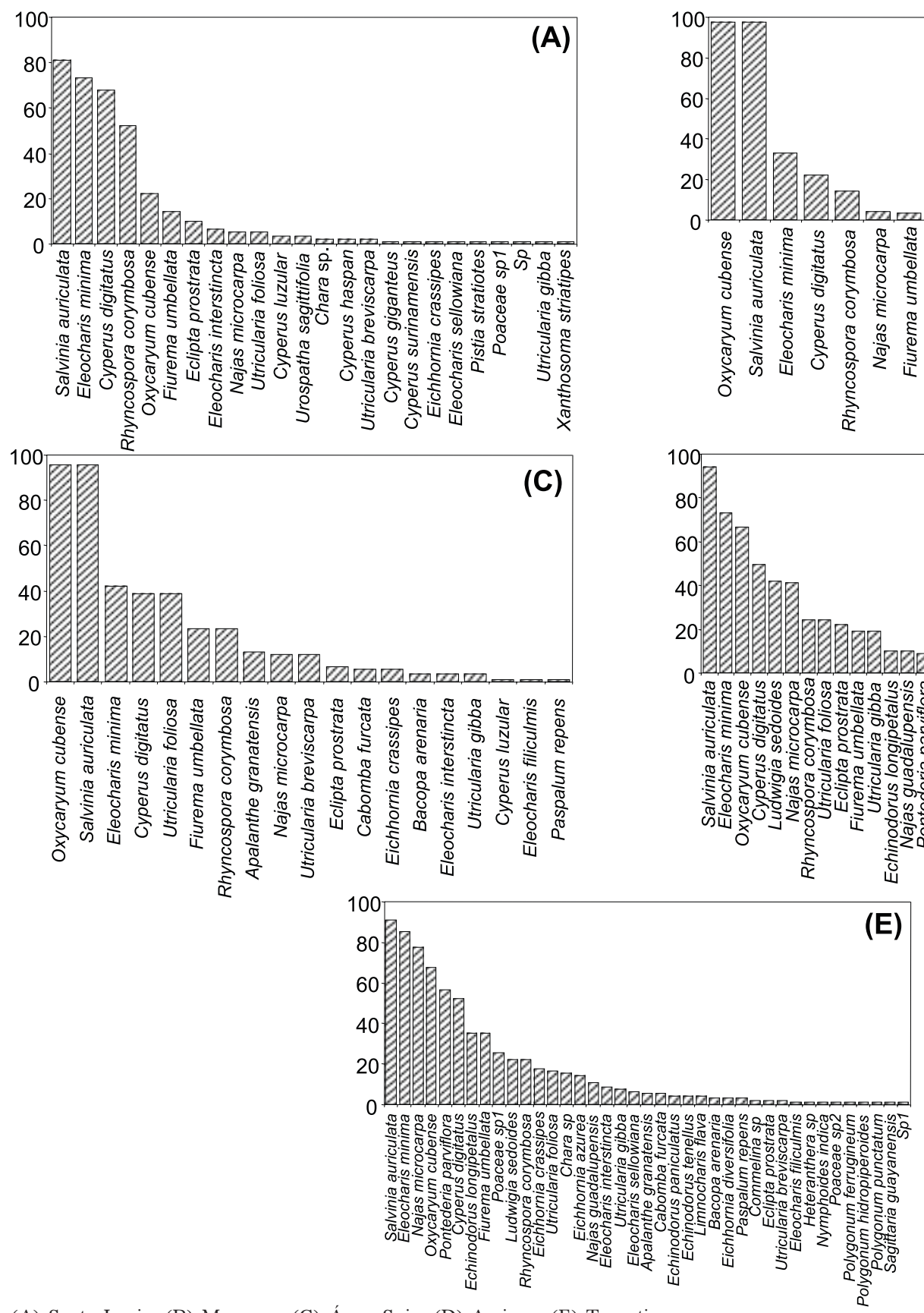

(A) Santa Luzia, (B) Mangues, (C) Água Suja, (D) Areias e (E) Tocantins.

Figura 5 - Valores da frequência de macrófitas em cinco regiões do reservatório.
Eleocharis minima, com frequência entre 33\% (Mangues) e 85\% (Tocantins), também foram espécies importantes em termos de ocorrência. Najas microcarpa destacou-se entre as espécies de maior frequência em Tocantins e Areias (77 e 41\%, respectivamente), enquanto Utricularia foliosa está entre as espécies mais frequentes na região de Água Suja (Figura 5).
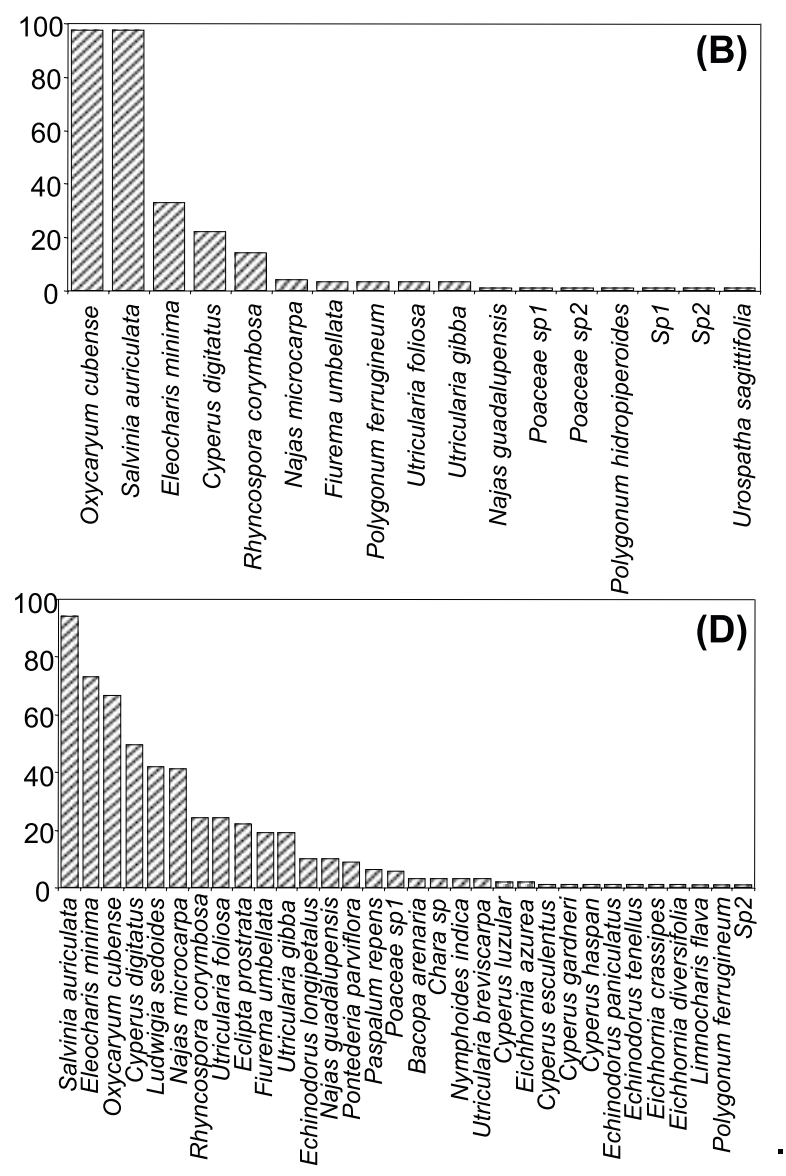

(E) 
A região com maior riqueza de espécies foi Tocantins (39 spp.), enquanto Mangues foi a que apresentou a menor riqueza (17 spp.). Houve diferenças significativas nos valores médios de riqueza entre as regiões $(\mathrm{F}(4$, 445) $=27,46 ; \mathrm{P}=0,001)$. Na verdade, houve aumento gradual nos valores médios ao longo do gradiente longitudinal do reservatório, com maiores valores nas regiões de montante (Figura 6).

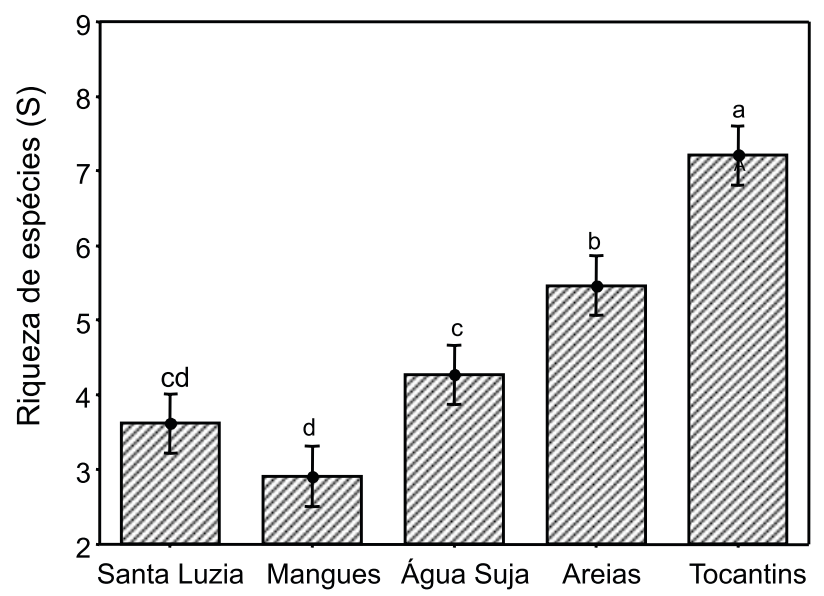

Letras iguais indicam ausência de diferenças significativas (Tukey; $\mathrm{P}<0,05$ )

Figura 6 - Valores médios e desvio-padrão de riqueza de espécies nas cinco regiões do reservatório.

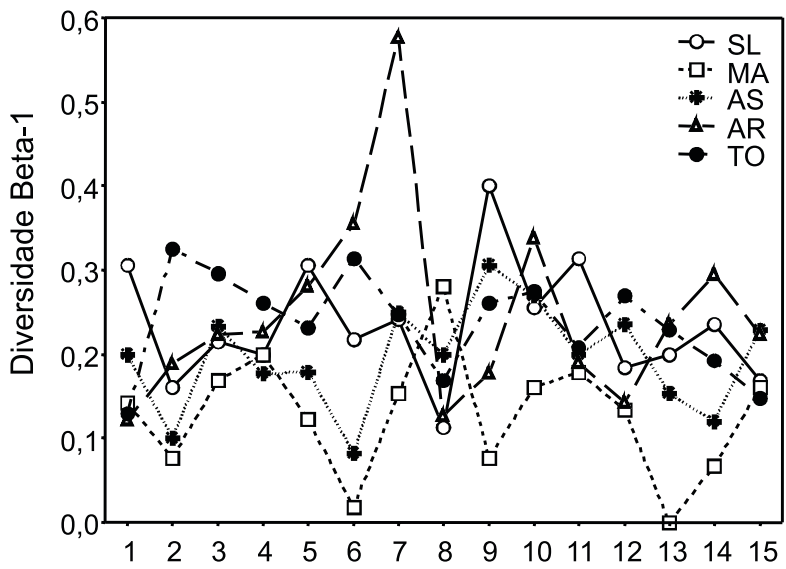

Adiversidade beta avalia a mudança ou taxa de substituição na composição de espécies, nas diferentes regiões amostrais (Whittaker, 1960; Harrison et al., 1992). Na presente análise, mediu-se a substituição temporal das espécies. A diversidade beta- 1 atingiu seu maior valor $(0,58)$ na região de Areias e o menor $(0,00)$ na região de Mangues. As demais regiões apresentaram valores intermediários (Figura 7A). A região que apresentou maior amplitude de variação foi a de Tocantins. A região de Mangues diferiu significativamente de Santa Luzia e Areias $(\mathrm{F}(4,57)=4,45, \mathrm{P}=0,003$ ) (Figura 7B).

O primeiro e segundo eixos da DCA retiveram $53 \%$ da variância total dos dados. Os escores da DCA mostraram que os eixos separam as regiões. As espécies que contribuíram positivamente para a formação do primeiro eixo foram $E$. minima e $N$. microcarpa, e negativamente, O. cubense e $S$. auriculata. Para a formação do eixo $2, R$. corymbosa e $C$. digitatus contribuíram positivamente, enquanto $O$. cubense e $N$. microcarpa foram as que mais contribuíram negativamente (Figura 8).

O primeiro eixo da DCA separou as regiões de Mangues, Água Suja e Tocantins, mostrando um gradiente longitudinal no sentido barragem. O segundo eixo separa claramente a região de Santa Luzia, que se localiza na região lacustre do reservatório. Os dados deste

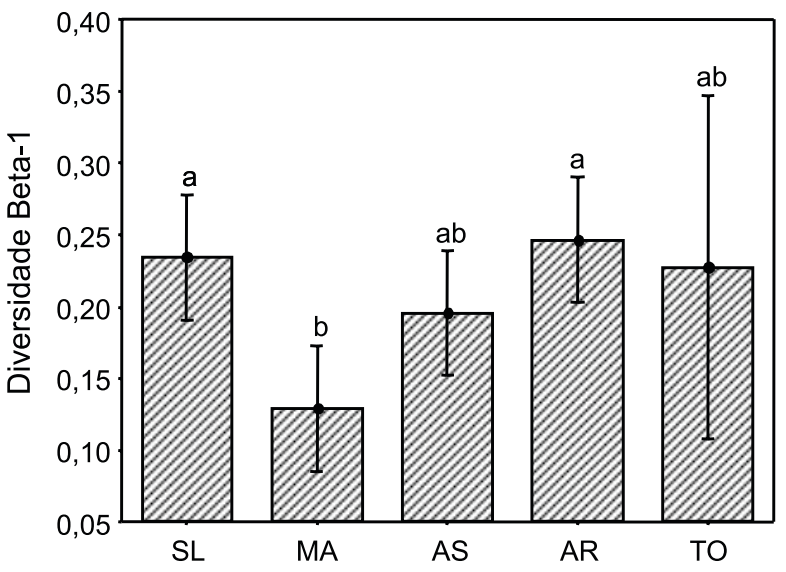

Letras iguais indicam ausência de diferenças significativas (Tukey; $\mathrm{P}<0,05$ ). $\mathrm{SL}=\mathrm{Santa}$ Luzia; $\mathrm{MA}=\mathrm{Mangues}$; $\mathrm{AS}=$ Água Suja; $\mathrm{AR}=\mathrm{Areias}$; e $\mathrm{TO}=$ Tocantins

Figura 7 - Variação na diversidade beta-1 dentro das regiões do reservatório, medida como a alteração da composição das assembleias em cada ponto de amostragem, ao longo dos meses (março/05 a janeiro/06) (A) e valores médios para as cinco regiões (B). 


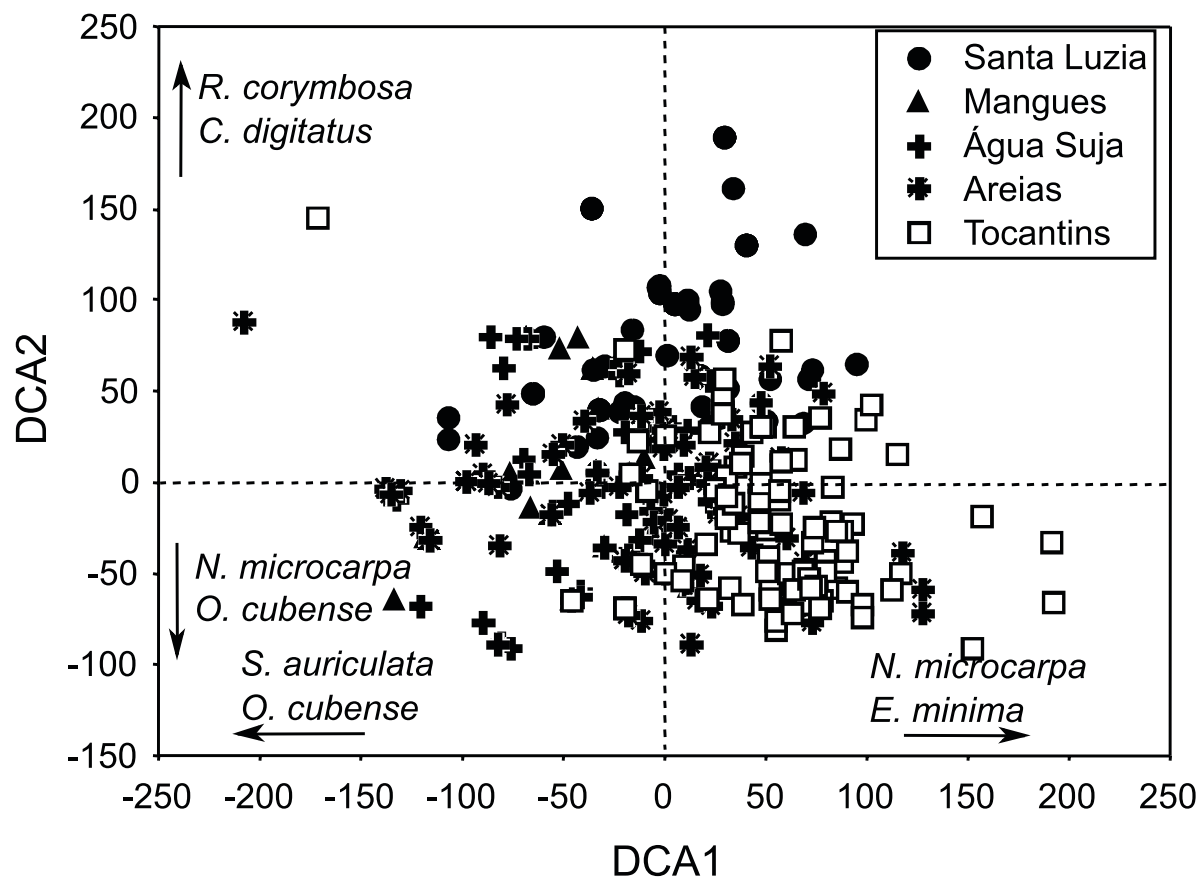

Figura 8 - Escores de uma análise de correspondência com remoção do efeito de arco (DCA), calculada sobre a matriz de presença/ ausência de espécies nas cinco regiões estudadas.

eixo mostram um padrão temporal, pois os meses de maio a setembro (período de seca) e de outubro a abril (período de chuva) mostramse diferenciados.

Os escores médios do eixo 1 diferiram estatisticamente entre as regiões do reservatório $(F(4,445)=99,2 ; P=0,001)$. Com exceção de Santa Luzia, houve aumento gradual na média dos escores ao longo do gradiente longitudinal do reservatório, indicando alterações progressivas na composição da comunidade em direção a montante (Figura 9A). Com relação à variação temporal nos escores, não houve diferença significativa na composição de espécies entre os meses amostrados $(\mathrm{F}(5$, 444) $=0,74 ; \mathrm{P}=0,59$ ) (Figura 9B).

Para os escores do segundo eixo, a região de Santa Luzia foi a única que diferiu estatisticamente $(\mathrm{F}(4,445)=63,8, \mathrm{P}=0,001)$ (Figura 10A). Esse eixo sumarizou certa variação temporal na composição das assembleias de macrófitas, com diferenças significativas a partir de maio $(F(5,444)=8,54, P=0,001)$ (Figura 10B).

\section{DISCUSSÃO}

Vários estudos têm demonstrado a rápida colonização por macrófitas aquáticas flutuantes em ambientes tropicais, como, por exemplo, Salvinia molesta no lago Kariba (Mitchell, 1969), Eichhornia crassipes, S. auriculata e Pistia stratiotes em um braço do reservatório de Itaipu (Thomaz et al., 1999) e S. molesta em um braço do rio Preto, Itanhaém (Rubim \& Camargo, 2001).

Nas cinco regiões estudadas no reservatório Lajeado, foi observada alta frequência de S. auriculata. Essa espécie é comum em ambientes aquáticos tropicais e tem ampla distribuição geográfica, sendo ausente em regiões frias (Boschilia et al., 2006). Em ambientes tropicais, ela apresenta alta taxa de crescimento e capacidade reprodutiva, principalmente dos módulos vegetativos (Thomaz, 2006). Observando a frequência temporal de S. auriculata no reservatório de Lajeado, verificou-se diminuição de aproximadamente 10\% em sua frequência, considerando o período de amostragem (março/2005 a janeiro/2006). Essa diminuição provavelmente está relacionada à redução das concentrações de nutrientes ao longo do tempo. Para o reservatório de Serra da Mesa-GO, no primeiro ano de operação foi estimada redução de $30 \%$ nas concentrações de nutrientes, o que foi atribuído à 

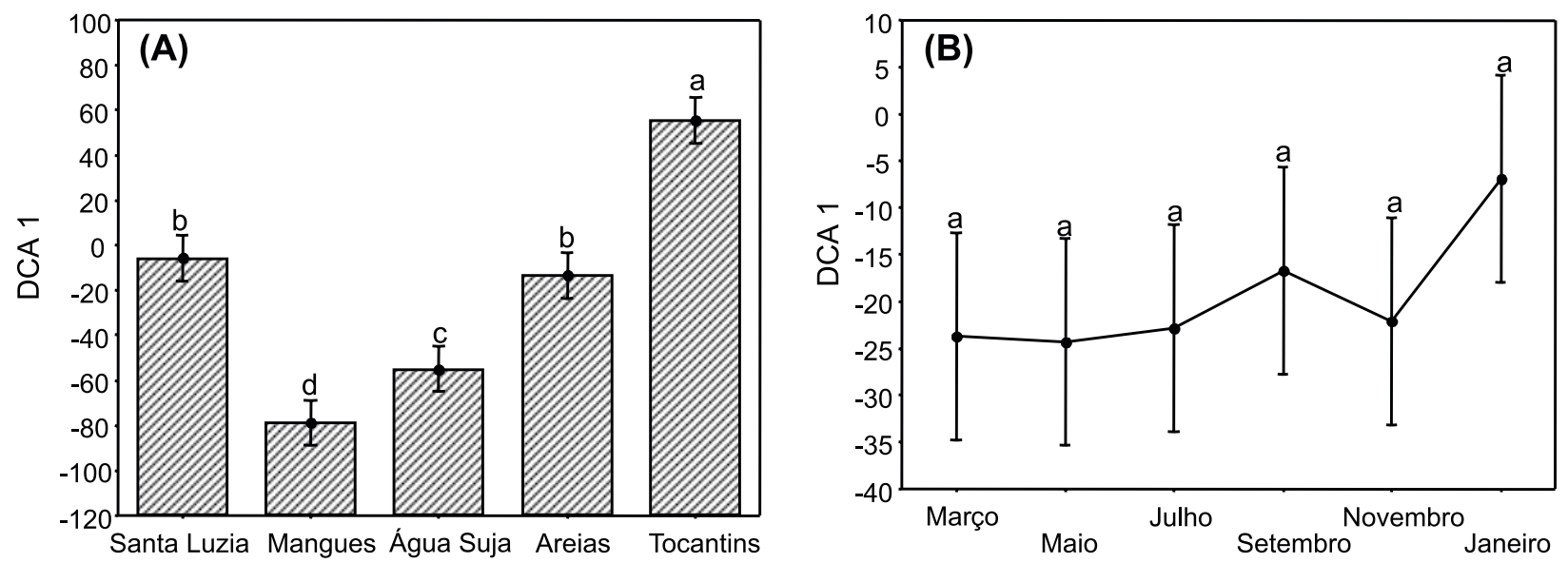

Letras iguais indicam ausência de diferenças significativas (Tukey; $\mathrm{P}<0,05$ )

Figura 9 - Valores médios e desvio-padrão dos escores do primeiro eixo da DCA, nas cinco regiões do reservatório (A), e a variação temporal considerando toda a área de estudo (B).
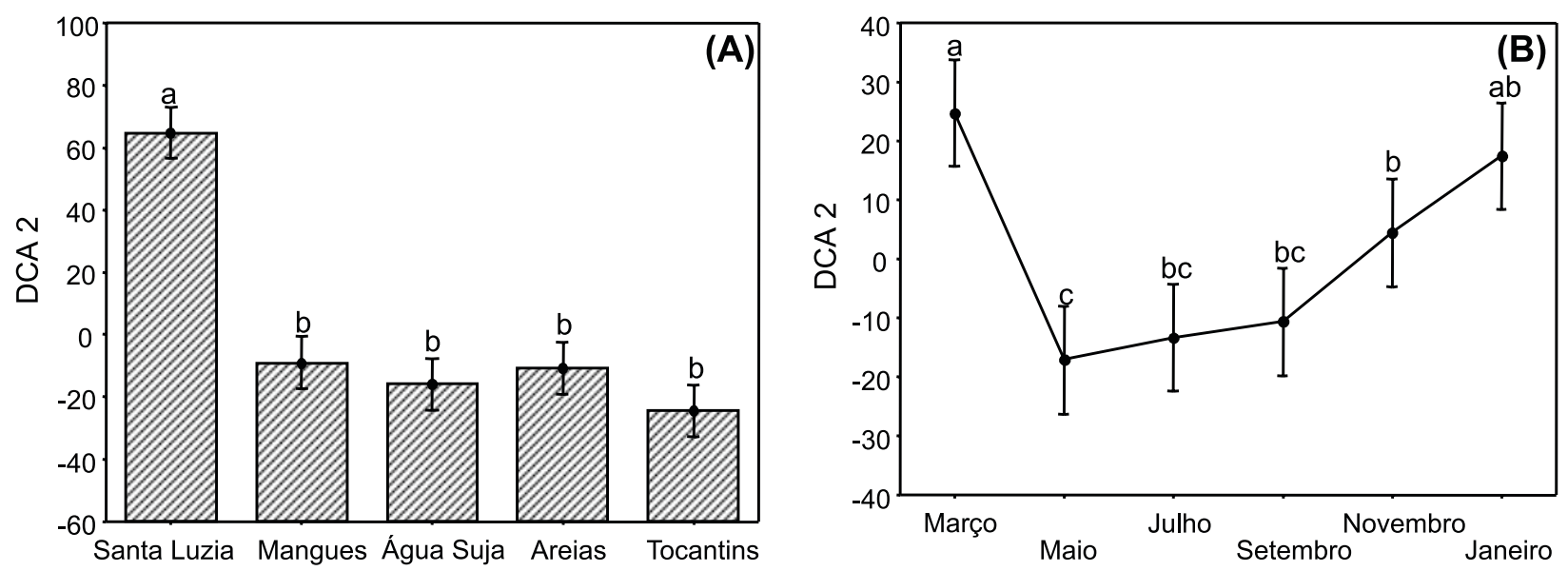

Letras iguais indicam ausência de diferenças significativas (Tukey; $\mathrm{P}<0,05$ ).

Figura 10 - Valores médios e desvio-padrão dos escores do segundo eixo da DCA, nas cinco regiões do reservatório (A), e a variação temporal considerando toda a área de estudo (B)

entrada de água dos rios e geração de energia (De Felippo, 2003). Esse mesmo autor também constatou diminuição na densidade de plantas aquáticas flutuantes nesse reservatório após a redução das concentrações de nutrientes.

O número de espécies variou entre as regiões amostradas no reservatório. Por exemplo, a riqueza de espécies foi mais elevada nas regiões de Areias e Tocantins, o que pode estar relacionado ao aporte de nutrientes, uma vez que as duas se encontram na região fluvial do reservatório. Thornton et al. (1990) ressaltam que, ao longo de um gradiente longitudinal, no sentido rio-barragem, ocorrem três zonas (fluvial, de transição e lacustre), com distintas características físicas, químicas e biológicas. $\mathrm{Na}$ região fluvial há maior aporte de nutrientes e carga de sedimento no reservatório, oriundos de tributários localizados a montante da barragem (Pagioro \& Thomaz, 2002). Nos trechos onde a taxa de sedimentação é maior ocorre redução da profundidade, propiciando a formação de áreas adicionais para colonização por macrófitas aquáticas (Thomaz et al., 2003). De fato, os aportes de sólidos são indicados pelos valores reduzidos de transparência nessa região (Figura 4E e F). 
O número de espécies submersas aumenta nas regiões onde a intensidade de radiação subaquática é maior, como na região do braço do Água Suja, onde foram registradas sete espécies submersas. A flutuação reduzida no nível do reservatório também favorece o desenvolvimento de uma comunidade de macrófitas submersas (Thomaz et al., 1999). Carvalho et al. (2003) realizaram levantamento das macrófitas aquáticas em cinco reservatórios localizados no rio Tietê e observaram que, naqueles com menores valores de turbidez, as principais infestantes foram as espécies submersas.

A maior heterogeneidade registrada em Areias é, provavelmente, resultado de sua localização na região fluvial do reservatório, onde ocorre maior taxa de sedimentação e áreas mais rasas são formadas, propiciando um ambiente adequado para a colonização de espécies de macrófitas aquáticas. Outro fator seria a grande área de vegetação alagada, onde a decomposição contribui para o aumento das concentrações de nutrientes.

De forma geral, os resultados obtidos neste estudo evidenciam que a variação espacial das assembleias foi mais importante que a variação temporal, conforme demonstrado pelos resultados da riqueza de espécies (Figura 6) e dos escores do primeiro eixo da DCA (Figura 9). A maior riqueza de espécies foi registrada nas regiões fluviais do reservatório (Areias e Tocantins). A riqueza de espécies foi mais elevada nos meses de março e novembro, que correspondem ao final e início do período chuvoso, respectivamente. Entre as variáveis abióticas consideradas neste estudo, a transparência da água parece ser a que mais contribui para o aumento das espécies submersas na região de Água Suja.

\section{AGRADECIMENTOS}

Ao Núcleo de Estudos Ambientais (NEAMB) da Universidade Federal do Tocantins e Núcleo de Pesquisa em Limnologia, Ictiologia e Aquicultura (Universidade Estadual de Maringá), pelas facilidades concedidas para coletas de campo e análises de laboratório; ao M.Sc. Rafael José de Oliveira (UFT), pelo auxílio nas análises estatísticas; a Dra. Elineide E. Marques, Dr. Carlos Sérgio Agostinho, Dr.
Fernando Mayer Pelicice (UFT), Dr. Fábio Amodeo Lansac Tôha e ao mestrando Roger Mormul (UEM Nupélia), pelas valiosas sugestões do manuscrito; à Investco, pelo auxílio financeiro para realização deste trabalho e pelos dados cedidos. S. M. Thomaz é bolsista de Produtividade em Pesquisa do CNPq e agradece a essa agência o constante apoio financeiro.

\section{LITERATURA CITADA}

AGOSTINHO, A. A.; GOMES, L. C. Reservatório de Segredo: bases ecológicas para o manejo. Maringá: EDUEM, 1997.

BIANCHINI JR., I. Modelos de crescimento e decomposição de macrófitas aquáticas. In: THOMAZ, S. M.; BINI, L. M. (Eds.). Ecologia e manejo de macrófitas aquáticas Maringá: EDUEM, 2003. p. 85-126.

BINI, L. M. et al. Aquatic macrophyte distribution in relation to water and sediment conditions in the Itaipu Reservoir, Brazil. Hydrobiologia, n. 415, p. 147-154, 1999.

BINI, L. M. et al. Patterns of the aquatic macrophyte cover in Cachoeira Dourada reservoir (GO-MG). Braz. J. Biol., n. 64, p. 767-770, 2005.

BOSCHILIA, S. M.; THOMAZ, S. M.; PIANA P. A. Plasticidade morfológica de Salvinia herzogii (de La Sota) em resposta à densidade populacional. Acta Sci. Biol., n. 28, p. 35-39, 2006.

CARVALHO, F. T. et al. Plantas aquáticas e nível de infestação das espécies presentes no reservatório de Barra Bonita, no rio Tietê. Planta Daninha, n. 21, p 105-108, 2003.

DE FELLIPO, R. Colonização e regressão da comunidade de macrófitas aquáticas no reservatório da UHE Serra da Mesa Goiás. In: THOMAZ, S. M.; BINI, L. M. (Eds.) Ecologia e manejo de macrófitas aquáticas. Maringá: EDUEM, 2003. p. 281-297.

HARRISON, S.; ROSS, S. J.; JAWTON, J. H. Beta diversity on geographic gradients in Britain. J. Ani. Ecol., n. 61, p. $151-158,1992$.

JUNK, W. J.; MELO, J. A. S. N. Impactos ecológicos das represas hidrelétricas na bacia amazônica brasileira. Est. Avançados, n. 4, p. 126-148, 1990.

MARTINS, D.; VELINI, E. D.; PITELI, R. A. Ocorrência de plantas aquáticas nos reservatórios da Light-RJ.

Planta Daninha, v. 21, p. 105-108, 2003. (Número Especial)

MITCHELL, D. S. The ecology of vascular hydrophytes on Lake Kariba. Hydrobiologia, n. 30, p. 448-464, 1969.

Planta Daninha, Viçosa-MG, v. 29, n. 2, p. 247-258, 2011 
NEIFF, J. J. et al. Prediction of colonization by macrophytes in the Yciretá reservoir of the Paraná river (Argentina and Paraguay. Braz. J. Biol., n. 60, p. 615-626, 2000.

PAGIORO, T. A.; THOMAZ, S. M. Longitudinal patterns of sedimentation in a deep, monomectic subtropical reservoir (Itaipu - Brazil-Paraguay). Archiv. Hydrobiol., n. 154, p. 515-528, 2002.

PAIVA, M. P. Grandes represas do Brasil. Brasília Editerra, 1982. $292 \mathrm{p}$.

PATTON, V. D.; STARNES, W. E. Aquatic weeds and water pollution. Hyacinth Control J., v. 8, n. 2, p. 48-49, 1970.

RIBEIRO, M. C. L. B.; PETRERE, M.; JURAS, A. A. Ecogical integrity and fisheries ecology of the AraguaiaTocantins Basin, Brazil. Regulated Rivers: Research \& Management, 1995; New York: John Wiley \& Sons, 1995

RUBIM, M. A. L.; CAMARGO, A. F. M. Taxa de crescimento especifico da macrófita aquática Salvinia molesta Mitchell em um braço do Rio Preto, Itanhaém, São Paulo.

Acta Limnol. Bras., n. 13,. p. 75-83, 2001.

TANAKA, R. H. et al. Ocorrência de plantas aquáticas nos reservatórios da companhia energética de São Paulo. Plantas Daninhas, v. 20, p. 101-111, 2002. (Edição Especial)

THOMAZ, S. M. et al. Aquatic macrophytes of Itaipu reservoir, Brazil: survey of species and ecological considerations. Braz. Arch. Biol. Technol., n. 42, p. 15-22, 1999.
THOMAZ, S. M.; BINI, L. M.; PAGIORO, T. A. Macrófitas aquáticas em Itaipu: ecologia e perspectivas para o manejo. In: THOMAZ, S. M.; BINI, L. M. (Eds.). Ecologia e manejo de macrófitas aquáticas. Maringá: EDUEM, 2003. p. $319-341$.

THOMAZ, S. M. Fatores que afetam a distribuição e o desenvolvimento de macrófitas aquáticas em reservatório: uma análise em diferentes escalas. In: NOGUEIRA, M. G.; HENRY, R.; JORCIN, A. (Orgs.). Ecologia de reservatório: Impactos potenciais, ações de manejo e sistema cascata. 2.ed. São Carlos: Rima, 2006. p. 165-181

THORNTON, K. W.; KIMEL, B. L.; PAYNE, F. E. Reservoir limnology: ecological perspectives. New York: John Wiley \& Sons, 1990. 248 p.

VALENTE, J. P.; PADILHA, P. M.; SILVA, A. M. M. Contribuição da cidade de Botucatu-SP com nutrientes (fósforo e nitrogênio) na eutrofização da represa de Barra Bonita. Ecletic. Quím., n. 22, p. 31-48, 1997.

VILARRUBIA, T. V.; COVA, M. Estúdio sobre la distribucion y ecologia de macrófitos acuaticos em el embalse de Guri. Interciência, n. 18, p. 77-82, 1993. <URL: http:/ www.interciencia.org.ve> Acesso em: 27 out. 2006.

WELCOMME, R. L. The fisheries ecology of floodplain rivers. London: Longman, 1979, $317 \mathrm{p}$

WHITTAKER, R. H. Vegetation of the Siskioy Mountains, Oregon and California. Ecol. Monog., n. 30, p. 279-338, 1960.

WILSON, M. V.; SHIMIDA, A. Measuring beta diversity with presence-absence data. J. Ecol., n. 72, p. 1055-1064, 1984. 